\title{
Spontaneous pneumothorax associated with primary lung cancer
}

\author{
Walid S Abu Arab*, Ahmed M Abdel-Aziz, Abdel-Maguid M Ramadan \\ From World Society of Cardiothoracic Surgeons 25th Anniversary Congress, Edinburgh \\ Edinburgh, UK. 19-22 September 2015
}

\section{Background/Introduction}

Spontaneous pneumothorax in association with primary lung cancer is infrequent. No exact mechanism is settled for its occurrence. However, it could be either a primary manifestation or complication for a primary lung cancer.

\section{Aims/Objectives}

The aim of this retrospective study is to search for the association of pneumothorax with primary lung cancer.

\section{Method}

Retrospective revision of the files of the patients who presented with spontaneous pneumothorax and admitted to the Cardiothoracic Surgery Department Main University Hospital of Alexandria, Egypt during the period from January 2005 to January 2015 was performed. Files were searched for association of spontaneous pneumothorax with primary pulmonary carcinoma. Patients who had spontaneous pneumothorax in association with pulmonary metastases were eliminated.

\section{Results}

Four patients have been found in our medical records. Two of them (50\%) had advanced squamous cell lung cancer (stage IIIB) and were referred to Oncology Department to receive treatment following pleural drainage with intercostal tube. One of the three $(25 \%)$ discovered the pulmonary carcinoma following surgery for recurrent spontaneous pneumothorax through bullectomy. This patient received formal lobectomy. Fourth patient had a bronchoalveolar carcinoma that discovered accidentally intra-operatively during bullectomy.

Cardiothoracic Surgery Department, Faculty of Medicine, University of Alexandria, Alexandria, Egypt 